Research Article

\title{
Finite Element Analysis of Air Flow and Temperature Distribution on Surface of a Circular Obstacle with Resistance and Orientation of Screen
}

\author{
Abid A. Memon $\left(\mathbb{D},{ }^{1}\right.$ M. Asif Memon $\mathbb{D}^{1},{ }^{1}$ Aisha M. Alqahtani $\mathbb{D}^{2},{ }^{2}$ Kaleemullah Bhatti $\mathbb{D}^{1},{ }^{1}$ \\ Kamsing Nonlaopon $\left(\mathbb{D},{ }^{3}\right.$ Ilyas Khan $\left(\mathbb{1},{ }^{4}\right.$ and Mulugeta Andualem $\left(\mathbb{1}^{5}\right.$ \\ ${ }^{1}$ Department of Mathematics and Social Sciences, Sukkur IBA University, Sukkur 65200, Sindh, Pakistan \\ ${ }^{2}$ Mathematical Sciences Department, College of Science, Princess Nourah bint Abdulrahman University, Riyadh, Saudi Arabia \\ ${ }^{3}$ Department of Mathematics, Faculty of Science, Khon Kaen University, Khon Kaen 40002, Thailand \\ ${ }^{4}$ Department of Mathematics, College of Science Al-Zulfi, Majmaah University, Al-Majmaah 11952, Saudi Arabia \\ ${ }^{5}$ Bonga University, Department of Mathematics, Bonga, Ethiopia
}

Correspondence should be addressed to Aisha M. Alqahtani; alqahtani@pnu.edu.sa and Mulugeta Andualem; mulugetaandualem4@gmail.com

Received 11 September 2021; Revised 16 October 2021; Accepted 18 October 2021; Published 8 November 2021

Academic Editor: Melike Kaplan

Copyright (c) 2021 Abid A. Memon et al. This is an open access article distributed under the Creative Commons Attribution License, which permits unrestricted use, distribution, and reproduction in any medium, provided the original work is properly cited.

\begin{abstract}
Nonisothermal flow through the rectangular channel on a circular surface under the influence of a screen embedded at the middle of a channel at angles $\theta$ is considered. Simulations are carried out via COMSOL Multiphysics 5.4 which implements the finite element method with an emerging technique of the least square procedure of Galerkin's method. Air as working fluid depends upon the Reynolds number with initial temperature allowed to enter from the inlet of the channel. The nonisothermal flow has been checked with the help of parameters such as Reynolds number, angle of the screen, and variations in resistance coefficient. The consequence and the pattern of the velocity field, pressure, temperature, heat transfer coefficient, and local Nusselt number are described on the front surface of the circular obstacle. The rise in the temperature and the flow rate on the surface of the obstacle has been determined against increasing Reynolds number. Results show that the velocity magnitudes are decreasing down the surface and the pressure is increasing down the surface of the obstacle. The pressure on the surface of the circular obstacle was found to be the function of the $y$-axis and does not show any impact due to the change of the resistance coefficient. Also, it was indicated that the temperature on the front circular surface does not depend upon the orientation of the screen and resistance factor. The heat transfer coefficient is decreasing which indicates that the conduction process is dominating over the convection process.
\end{abstract}

\section{Introduction and Literature Review}

When any type of fluid having some initial velocity and the temperature is coming to strike with a certain surface especially the circular surface, it transmits the heat/energy (depending upon the velocity and several factors) on the surface at a certain pattern of behavior or with the certain amplitude [1]. For optimization of the temperature and explaining the behavior of the fluidic and thermal parameters, in the current problem, we are going to use the screen settled at a certain angle, coefficient resistance, and Reynolds number. The nonisothermal flow through the circular surface had been studied extensively for few decades. All studies were focusing on maximizing the temperature distribution over the surface by changing of used parameters in the problems $[2,3]$. The heated circular obstacles are widely used in the application of generation powers, thermal equipment, cables, nuclear apparatus, and heat distributors [4-6]. Many researchers and scientists put on the lights on the study of fluid flow and heat transfer in the presence of circular obstacles, few of them described as follows. 
A nonisothermal flow of air around the circles was studied [7] with the range of the Reynolds number from 40 to 10,000 by putting the Prandtl number at 0.7 with the use of commercial software FLUENT. The circles were arranged as in-line and staggered and suggested that the heat transfer is optimized when the circles are arranged in a staggered pattern. A numerical investigation and the tunnel experiment [8] were done by arranging in-line cylinders with circular and elliptical orbits using different ranges of the Reynolds number. Based on the Nusselt number, it was concluded that using any shape can be used as the heat exchanger but cylinders with the elliptical shape are more efficient. Through the circular cylinder and using the flexible fin, heat transfer via convection was studied numerically by [9] with a unique $\operatorname{Re}=200$ and $\operatorname{Pr}=0.7$. They employed the Galerkin finite element method and the semitorsional analogy method to solve the Navier-Stokes equations coupled with the energy equation and the Bernoulli beam equation. It was found that with the vibration of the fin, a vortex shedding is induced behind the cylinder, and with the increase in vibration of fin, convection heat transfer is improved further. An experiment [10] was performed to measure the heat transfer and the pressure drop through the channel embedded by the hollow circles in the range of the Reynolds number from 6000 to 27,000 with 2 as a perforated ratio. They measured that the convective heat transfer is dominated over the conduction, i.e., the global Nusselt number, and the friction factor is increased with the increase in pitch ratio. Most scientists are thinking that the constant thermal boundary conditions due to forced convection in the tube with a circle do not affect the heat transfer. A study [11] was performed with the range of the Reynolds number $\operatorname{Re}<3000$ to reject the claim. With the findings of the different fluid and thermal parameters, they rejected the claim. A study [12] of the fluid flow behavior and the heat transfer through the circular tube was done by introducing the new drag coefficient composed of the ratio between the Nusselt number and the Prandtl number. It was suggested that there may be an authentic but strong relation that exist between the new drag coefficient, mass, and heat transfer, and the relation provided in the study may be crucial due to the lack of information on the Nusselt and Sherwood number. The computational and theoretical study [13] was done for the understanding of the heat transfer with free convection and fluid flow on the outer surface of circular cylinder present under the electro-conductive polymer solution. It was found that with the increase in the Weissenberg number, there is a very little increment in the temperature but the flow is gradually accelerated near the circular cylinder. Arranging many cylinders in the horizontal pattern [14], a new correlation was determined for the heat transfer in the natural as well as forced convection processes with extensive analytical studies. The implications of the VonKarman-Pohlhausen numerical approach $[15,16]$ analysed the fluid flow and the heat transfer on the front surface of the cylinders. The methodology was tested for the single as well as putting infinite cylinders in the domains. Tremendous research was done, and to use a wide range of the
Reynolds numbers and Prandtl number, a correlation formula was found between heat transfer and the drag coefficients. The effects of pressure and drag coefficients due to the variation of longitudinal distances between the two circular cylinders were determined by several experiments $[17,18]$ in the case of a long-range Reynolds number. An experiment with the extensive study was performed by [19] to investigate the local pressure coefficients and the local Nusselt number on the surface of circular cylinder for the various Reynolds numbers under variation in several the blockage ratios. Later on, this study was used to investigate the fluid flow and heat transfer for the tube banks of the cylinders. The rate of cooling for the circular cylinder of copper with an experiment was performed by [20] with the forced convection procedure. The author has also investigated the uncertainty for characteristics of thermal distribution in the system. With the use of a $\kappa-\omega$ model, a numerical time-dependent and independent study was done to determine the heat transfer and fluid flow through a cylinder for crossflow [21]. It was found that vortex shedding can only be observed with the time-independent study. On analysing the power-law fluid of Newtonian with the high Reynolds number under the influence of screen [22], it was found that on the surface of a circular cylinder, the ratio of output speed to the input speed of flow remains constant and the ratio increases with the increase in power-law index " $n$." Also, it was seen that with altering the angle of the screen, there was no significant change in the thermal performance on the surface of the circular cylinder. The use of Galerkin's scheme of the finite element method for the fluid flow passed on the semicircular cylinder was investigated [23] with the range of the Reynolds number from 100 to 1500 . The research throws the light on a horizontal and vertical pattern of flow of the velocity field as well as pressure, and it was explained that the two vortices and the back of the cylinder are produced whose length is the function of the Reynolds number. The fluid flow parameters and the behavior of the fluid under the influence of magnetic field on the surface of the circular cylinder have been studied [24] with the ratios of the rectangular channel to the diameter of the circle between 1.6 and 4 and the Stuart number less than 5. They deducted that the recirculation region was seen at the end of the cylinder and suggested a critical Stuart number to keep the drag on the surface of the cylinder lower. The behavior of the velocity field on the circular obstacle fixed in the cavity has been determined by [25] with the implementation of the multigrid technique of the finite volume method. A recirculation appeared at the back of the cylinder, and the length of that recirculation is increased with the increase in flow velocity. With the finite volume approach, the fluid flow characteristics along with heat transfer were determined by [26] when the fluid is coming to strike with the circular obstacle fixed in a rectangular channel keeping $\mathrm{Re}$ in the range of 50-180. It is concluded that the temperature distribution, lift force, and drag force show the direct response with an enhancement of the Reynolds number. An emerging technique [27] was developed known as Galerkin's least square procedure to 
solve all types of fluid flow and heat transfer problems relating to compressible and incompressible fluids. According to their suggestion, if the Navier-Stokes equations are assumed as the quasilinear form, then a general formulation can be derived through the finite element method to get the entropy variables velocity, temperature, and pressure. The fluid flow through the screen attached to the middle at an angle was presented by [28] to give the streamwise velocity at the outlet of the channel. The solution is known as an asymptotic solution and gives a brief introduction of the characteristic of the airflow under the impact of the screen through the rectangular channel. With the implementation [29] in of FEM technique, the working fluid air was tested to optimize the velocity and drag force under the influence of three screens arranged from -45 degrees to 45 degrees. The whole process was simulated on the COMSOL Multiphysics 5.4, and results were substantiated with the available literature. It was deducted that reckoning more screens in the channel optimizes the velocity as well as drag force on the channel. Newtonian as well as non-Newtonian observation [30] of the airflow through the perforated plates was observed by fixing the power-law index from 0.7 to 1.3 and the Reynolds number from 1000 to 10,000 using the finite element package of COMSOL Multiphysics 5.4. The results showed great similarity with those present in the literature, and a parametric study was performed to have a clear idea of dependence of velocity profile and pressure upon Reynolds number and power-law index. With the use of the screen boundary conditions on the solid fixed at the middle of the channel, a study [31] was done with altering the resistance coefficient from 2 to 3 and inlet velocity from $0.2 \mathrm{~m} / \mathrm{sec}$ to $1 \mathrm{~m} / \mathrm{sec}$ to maximize the flow rate at the exit of the channel with the method of Galerkin's scheme. It was shown that the speed of the fluid particles also increases by increasing the resistance coefficient of the screens. In this way, many of the applications of linear and nonlinear sciences can be seen for the observed problems $[32,33]$ when one tried his best to achieve the analytical and numerical solution for the governing partial differential equations in the respective fields and areas.

In the current problem, we are going to focus on the fluid flow behavior and heat transfer to the surface of the circular obstacle in the presence of the screen arranged for $\pi / 6, \pi / 4$, and $\pi / 3$ with the Reynolds number from 1000 to 10,000 using the different coefficients of the resistance of the screen from 1 to 3 . The results are gained through Galerkin's least square scheme of the finite element method, and the whole procedure was taken on the COMSOL Multiphysics 5.4. The problem is very new because here we are observing the fluid flow and heat transfer on the circular obstacle in the presence of a perforated screen with the addition of the orientation of the screen as well as resistance coefficients. The results are presented through graphs, and the empirical equation is derived for the change in velocity from inlet to the surface of the circular obstacle. In the problem, we intend to give the optimum solution for both the velocity field, temperature, and the pressure of the fluid related to the parameter used in the current problem.

\section{Methodology}

2.1. Description of the Channel. The selected geometry in the channel is rectangular with the observed ratio of $4: 1$ from length to height of the channel. A circular obstacle with a radius of $0.25 \mathrm{~m}$ is embedded at the end of the channel so that the aspect ratio between the height of the channel to the diameter of the channel is 1: 1 . A screen with the variable resistance coefficients $\kappa$ from 1 to 3 is fitted at the middle of the channel which is capable of moving the angles of $\pi / 6$, $\pi / 4$, and $\pi / 3$ radians (see Figure 1 ). The angles of the screen are measured in the clockwise direction from the vertical line at the center of the channel. A laminar, steady-state, and Newtonian fluid flow has been tested along with the heat transfer for the channel. For the laminar flow, an average velocity depending on the Reynolds number is set to allow from the inlet of the channel and for the heat transfer initial temperature of $293.15 \mathrm{~K}$. The upper and lower walls of the channel are perfectly insulated with slip boundary conditions to ignore the viscous effects at the walls. However, the circular obstacle which will be under observation for the thermal and dynamical process is not perfectly insulated, but a heat flux $q w=10 w / \mathrm{m}^{2} \mathrm{~K}$ is applied to calculate the heat transfer coefficient and local Nusselt number on the front surface of it.

2.2. Meshing of Geometry. It is the beauty of the several numerical methods to solve the physical problems governed through the nonlinear partial differential equations and even in the case of a coupled system to divide the whole domain of observation into small pieces called elements. In the first step, the governing partial differential equations are converted into a system of algebraic equations with the weighted residual procedure. Then, those sets of algebraic equations are solved for each element. Definitely, the larger the number of elements, the procedure will become the time taken and the convergence does matter for the problem. In the present problem, we have divided the whole domain of interest into small irregular triangles (see Figures 2(a) and 2(b)). In Figure 2(a), we have placed the view of coarse mesh; in Figure 2(b), we used the fine mesh. Further, we have used the more fine mesh where we find the case is more sensitive, for example, near the circular obstacle. A total of 2412 triangular elements were used in different areas and quality. The mesh statistics are given in Table 1.

\subsection{The Governing Equations of Fluid Flow and Heat} Transportation. The set of partial differential equations that describe the motion of the fluid particles while undergoing through any medium and possessing the viscous effects is known as Navier-Stokes equations. Using the law of conservation of momentum and the law of conservation of mass, the Navier-Stokes equations are derived by Claude-Louis Navier and George Gabriel Navier with the assumption that the total stress of the fluid can be summed as the gradient of the velocity field (diffusing viscous). The equation is used to describe the motion of the particles containing velocity field, pressure, viscosity, and density terms. Moreover, the 


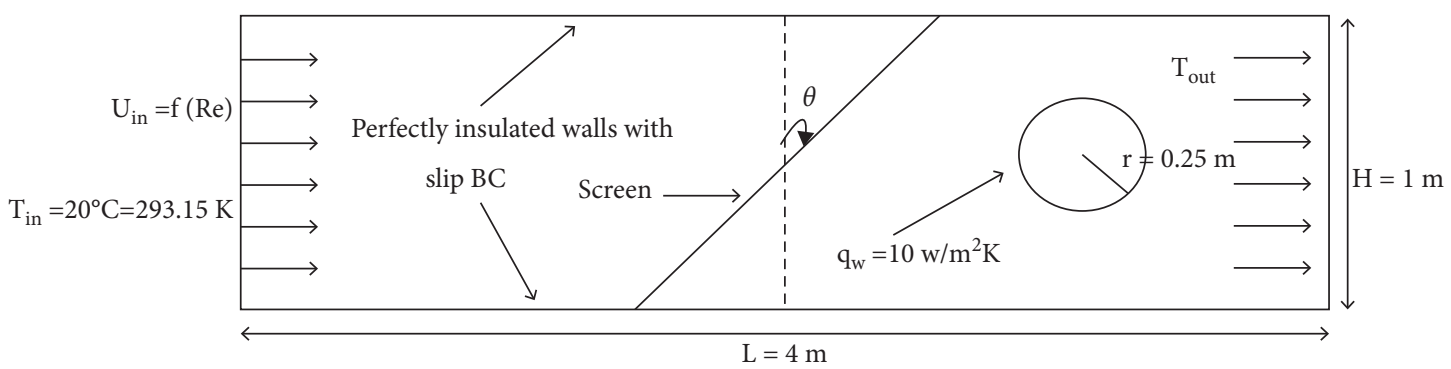

Figure 1: Structure of the rectangular medium under observation [16].



(a)

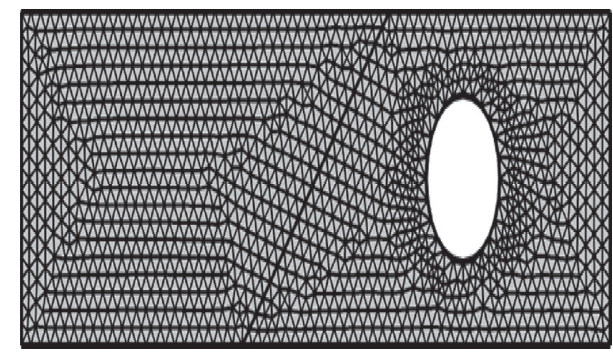

(b)

Figure 2: (a) Irregular triangular coarse mesh. (b) Irregular triangular fine mesh [16].

TABLE 1: Mesh statistics of simulation.

\begin{tabular}{lc}
\hline Properties & Value \\
\hline Mesh vertices & 1294 \\
Triangles & 2412 \\
Edge elements & 197 \\
Vertex elements & 10 \\
Min: element quality & 0.5328 \\
Average element quality & 0.8997 \\
Element area ratio & 0.1989 \\
Mesh area & $3.806 \mathrm{~m}^{2}$ \\
\hline
\end{tabular}

Navier-Stokes equations are parabolic and possess strong analytic properties.

In the present problem of the nonisothermal flow, we are going to simulate the laminar flow and the heat transfer through the rectangular channel attached by the screen and a circular obstacle at the end of the channel. The problem is steady-state and momentum equation along with the continuity equation which is discretized by the least square procedure of the finite element method. The momentum and continuity equation is given as follows:

$$
\begin{aligned}
\rho(U . \nabla) \cdot U & =\nabla[-p I+K]+F, \\
\rho(\nabla . U) & =0, \\
K & =\mu\left(\nabla U+(\nabla U)^{T}\right),
\end{aligned}
$$

where $\rho$ is the density of the fluid, $p$ is the pressure, $K$ is the stress tensor, and $U$ is the velocity field.

In our case, the upper, lower, and surrounding of the circular obstacles are not going to take part to visualize the viscous effects because we need to view the distribution of the heat and the dynamical parameters like velocity and the pressure on the walls. For this purpose, we like to force slip boundary conditions on all upper and lower walls which implies that these walls will be restricted to leave the domain at any instant. We can define the slip boundaries as follows:

$$
\begin{aligned}
U . \mathbf{n} & =0, \\
-p I+\mu\left(\nabla U+(\nabla U)^{T}\right) n & =0 .
\end{aligned}
$$

Here is the normal vector on the velocity field.

In our problem, to optimize the temperature and to compare with the available literature, the screen boundary condition is used to fix a solid screen at the middle of the channel. Screens are widely used from the ancient for the purpose to move the direction of the flow to the favorite one. The main functions of the screen are to suppress the horizontal component of the velocity field and make a resistance to create kinetic energy relating to turbulence. We can define the screen boundary condition as follows:

$$
\begin{aligned}
{[\rho U . \mathbf{n}] } & =0 \\
{\left[p-n^{T} \mathbf{\kappa} \mathbf{n}+\rho(U . \mathbf{n})^{2}\right]_{-}^{+} } & =-\frac{\kappa}{2} \rho_{-}(U . \mathbf{n})^{2}, \\
\mathbf{n} \times U_{+} & =\eta\left(\mathbf{n} \times U_{-}\right) .
\end{aligned}
$$

In equations (3)-(5), $\kappa$ is the resistance coefficient ranging in the current simulation from 1 to 3 and $\eta$ is the refraction coefficient and fixed for the current simulation with 0.78. "+" and "- " subscriptions show the direction of the flow before screen (upstream) and after screen (downstream) in equations (3) to (5). 
The heat equation is widely used to predict the temperature distribution in the region of interest. Especially, it is recognized as the diffusion equation. The heat equation is widely studied in the context of the partial differential equation because of the resemblance with so many other constitutive equations like Black Scholes used in finance. It is also used to understand the random walks like Brownian motion with the adding of the certain. In the problem under consideration, we are going to optimize the temperature distribution in the rectangular plate under the influence of the orientation of the screen, Reynolds number, and the resistance coefficients. The heat equation is given as follows:

$$
\begin{aligned}
\rho C_{p} U . \nabla T+\nabla \cdot q & =Q, \\
q & =-\kappa \nabla T,
\end{aligned}
$$

where $C_{p}$ is the specific heat capacity (J/kg.K) at the constant temperature, $T$ is the absolute temperature $(\mathrm{K}), q$ is the heat flux by conduction $\left(\mathrm{W} / \mathrm{m}^{2}\right), \kappa$ is the conductive heat transfer coefficient $\left(\mathrm{W} / \mathrm{m}^{2} \mathrm{~K}\right)$, and $Q$ is the heat source $\left(\mathrm{W} / \mathrm{m}^{2}\right)$.

An average velocity $U_{\text {in }}=f(\mathrm{Re})$ is allowed to enter from the inlet of the rectangular channel with the initial temperature $T_{\text {in }}=20^{\circ} \mathrm{C}=293.15 \mathrm{~K}$. On the walls, slip boundary conditions are applied and the walls are perfectly insulated $(-\mathbf{n} \cdot \mathbf{q}=0)$ so that the heat cannot transfer across the boundaries. A circular obstacle is surrounded by the heat flux condition of $q_{w}=10 \mathrm{~W} / \mathrm{m}^{2}$. After getting the results for the velocity field, pressure, and temperature distribution, we go to collect the information about the heat transfer coefficient and the local Nusselt number by the following relations on the front surface of the circular obstacle. Here, we can describe the equations as follows:

$$
h=\frac{q_{w}}{T-\mathrm{Tb}},
$$

where $\mathrm{Tb}$ is the bulk temperature and can be evaluated as follows:

$$
\frac{\int_{0}^{H} U(x, y) T(x, y) \mathrm{d} y}{\int_{0}^{H} U(x, y) \mathrm{d} y} .
$$

And after the heat transfer coefficient, we would calculate the local Nusselt number by the relation as follows:

$$
N \mu_{x}=\frac{h x}{\kappa} \text {. }
$$

\section{Validation and Comparison}

Before going to discuss the results obtained for the simulation of the nonisothermal flow on the surface of the obstacle, we are going to validate our result by comparing the streamwise velocity at the outlet of the channel provided by the Elder [1959] who found the asymptotic solution by attaching the screen at an angle $\theta$ at the middle of the rectangular channel and suggested the following relation.

Here, $U$ is computed numerically with Galerkin's least square procedure in the current simulation through the
COMSOL Multiphysics 5.4. The expression in the equation either the left-hand side or right-hand side is called the streamwise velocity. In Figure 3(a), the streamwise velocity is computed with the screen but without the circular, obstacle at the outlet of the channel, and we found that our simulation shows good agreement with the asymptotic solution. On computing the streamwise velocity with both screen and the circular obstacle, the asymptotic solution shows good agreement with negligible error with the numerical computation (see Figure 3(b)).

\section{Result and Discussion}

The present problem for the nonisothermal flow through the rectangular channel with the fixed circular obstacle at the end of the channel has been solved with Galerkin's least square scheme of the finite element method, and the whole procedure of simulation was carried on the commercial software COMSOL Multiphysics 5.4. The fluid flow was tested by the parametric study of the Reynolds number from 1000 to 10000 , keeping the resistance coefficient $\kappa$ of the screen from 1 to 3 , and the screen is movable with the specific angles for $\pi / 6, \pi / 4$, and $\pi / 3$ radians. For the problem, we are going to present the results through interpolated graphs for the velocity magnitude, pressure, temperature, and heat transfer coefficient at the measurement of the local Nusselt number at the front surface of the circular obstacle by fixing any two of the three parameters Re and $\kappa$ and angle of the screen. We are also going to describe the results for the change in the velocity magnitude as well as the change in the temperature of the fluid from the inlet to the surface of the channel by fixing the resistance coefficient $\kappa$ against the variable Reynolds number. Though the front surface of the circular obstacle is the vertical measurement, we intend to present the results along the $y$-axis only. We found that our chosen dynamical parameters like velocity and pressure are the functions of the vertical measurements. The current problem will help the reader to understand the dynamics and heat transfer on the surface of the circular obstacle and also to optimize any of the dynamical parameters with the help of Re, resistance coefficient $\kappa$, and angles of the screen.

In Figures 4(a) and 4(b), we have presented the graphs for the orientation of the velocity magnitude $(\mathrm{m} / \mathrm{sec})$ against the $y$-axis for the front surface of the obstacle. The front surface is composed of two parts, below the central line ranging $(0<y<0.5)$ and above the centerline $(0.5<y<0.8)$. In Figure $4(a)$, the pattern of the velocity field indicates that the flow rate of the fluid particles is decreasing down the surface of the circular obstacle and increasing the velocity in magnitude above the surface by fixing $\mathrm{Re}=1000$ and $\kappa=1$. Although by altering the angle of the screen, the flow rate is nearly consistent on the front surface of the obstacle. In Figure 4(b), the pattern of the velocity field has been checked by changing the Reynolds number from 1000 to 10,000 at a fixed angle $\pi / 6$ and $\kappa=1$. The graph indicates that the behavior of the velocity field is the same but the flow rate of the fluid particles is increasing with the increase in the Reynolds number by fixing the angle and resistance of the screen. It is obvious that the flow rate of the particles is 


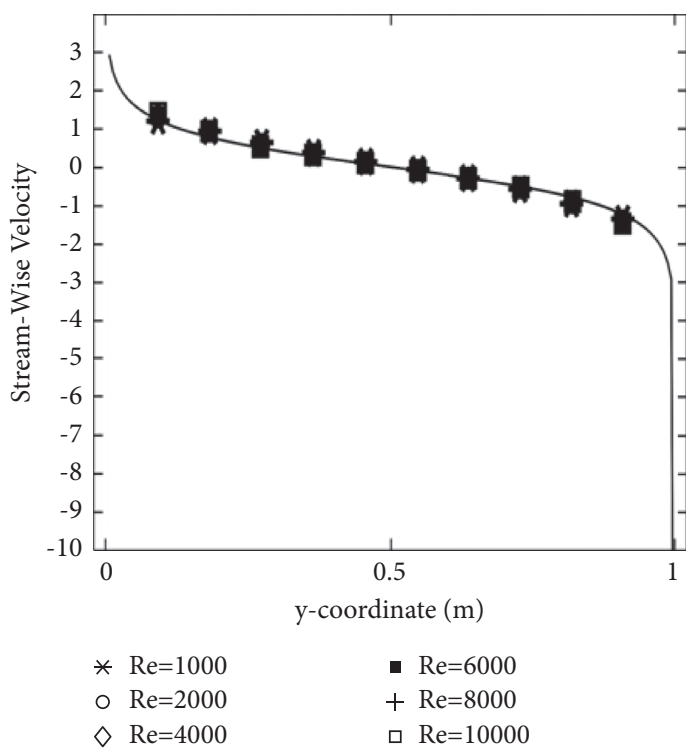

(a)

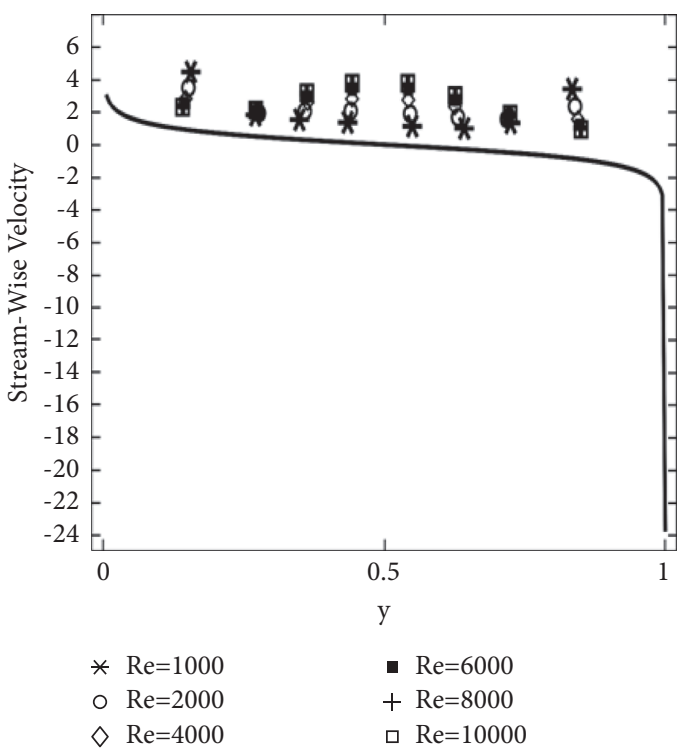

(b)

FIgure 3: (a) Comparison of the asymptotic solution with the numerical solution in presence of screen only. (b) Comparison of the asymptotic solution with the numerical solution in the presence of screen and circular cylinder.

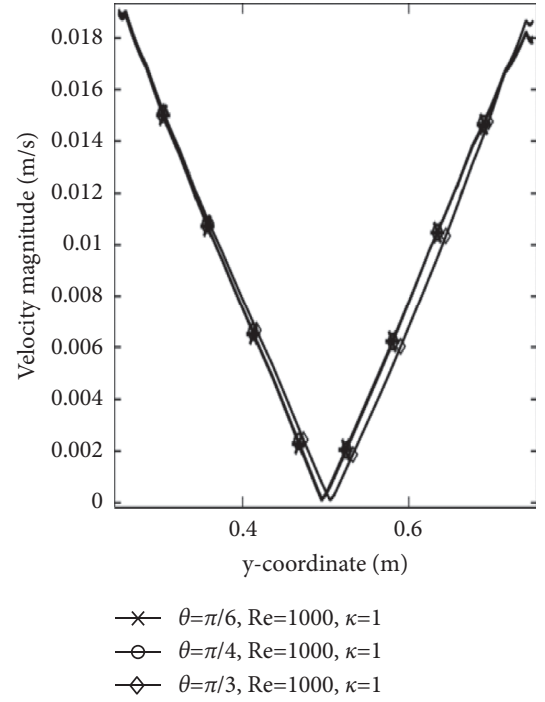

(a)

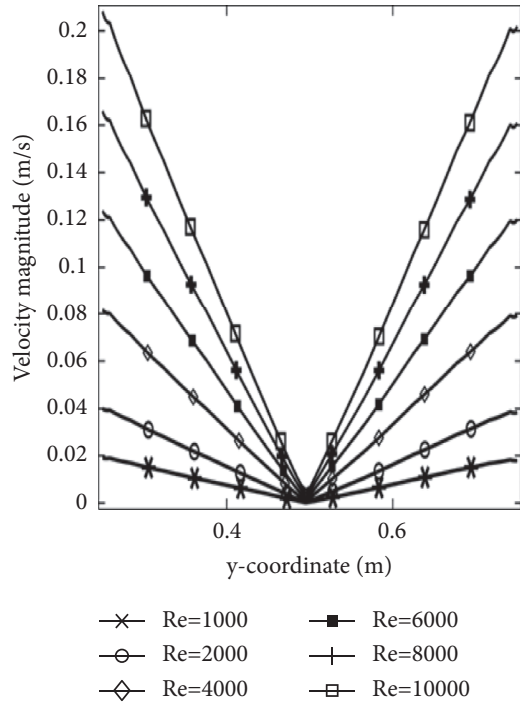

(b)

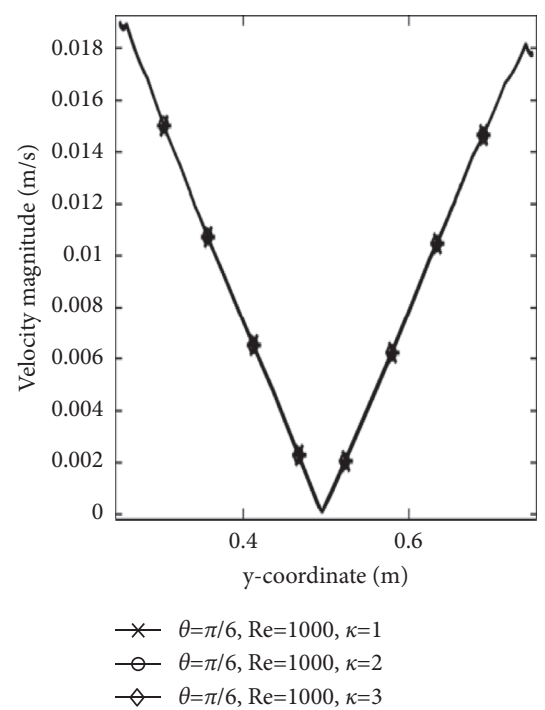

(c)

Figure 4: Pattern of the velocity magnitude (m/sec) on the front surface of the circular cylinder (a) for all angles by fixing Re $=1000$ and $\kappa=1$; (b) for all $\operatorname{Re}$ by fixing $\kappa=1$ and $\theta=\pi / 6$; (c) for all $\kappa$ by $\operatorname{Re}=1000$ and $\theta=\pi / 6$.

linearly increasing or decreasing in the front surface of the obstacle and is the function of the $y$-axis. In Figure $4(\mathrm{c})$, the velocity magnitude of the particle on the front surface is presented by fixing the angle of the screen at $\pi / 6$ and $\mathrm{Re}=1000$ with the increase in the resistance of the screen from $\kappa=1$ to $\kappa=3$. It depicts that the observation fails to change the pattern of the velocity field. Thus, altering the facing resistance of the screen does not give a significant increment in the case of velocity magnitude for fixing the Re and angle of the screen. It is added that all the points having zero velocity divide the surface into two parts. This is the actual location where the small vortex may appear.

In Figures 5(a)-5(c), the pressure $(\mathrm{Pa})$ is plotted against the $y$-axis on the front surface of the circular obstacle by fixing the parameters. The graphs indicate that the striking of the fluid on the front surface yields both the positive pressure and the negative pressure above or below the front surface. Such a property of the flow allows the fluid to attract and contract in certain directions. In Figure 5(a), the pressure is plotted for 


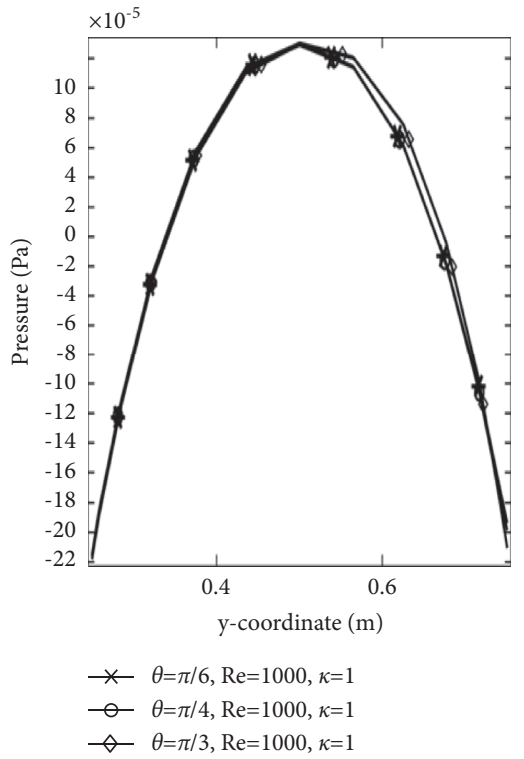

(a)

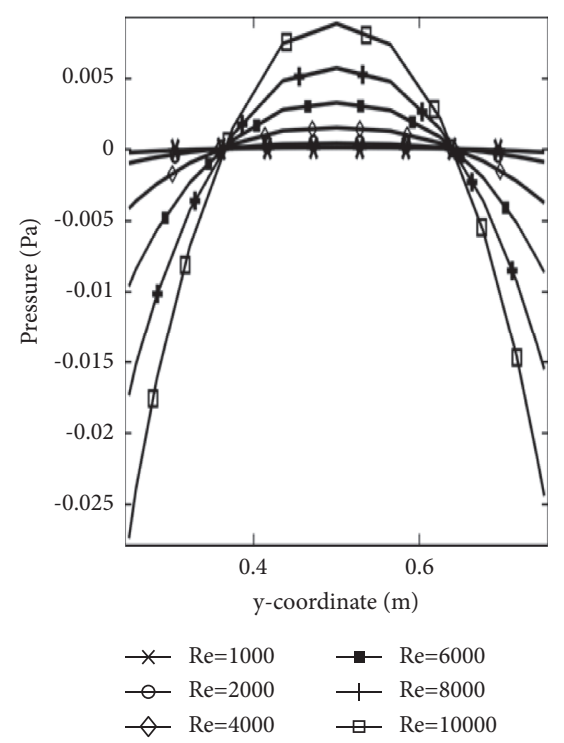

(b)

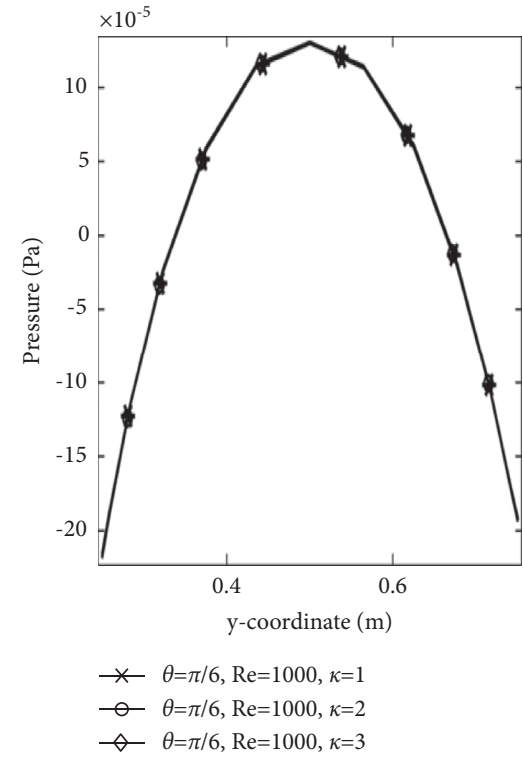

(c)

Figure 5: Pressure $(\mathrm{Pa})$ description on the front surface of the circular cylinder (a) for all angles by fixing $\mathrm{Re}=1000$ and $\kappa=1$; (b) for all Re by fixing $\kappa=1$ and $\theta=\pi / 6$; (c) for all $\kappa$ by $\operatorname{Re}=1000$ and $\theta=\pi / 6$.

varying the angle of screens with the fixing of the Reynolds number at $\operatorname{Re}=1000$ and $\kappa=1$. The graphs show that the pressure is maximum at the middle of the surface. However, changing the angle of the screen does not impact pressure insignificantly. In Figure 5(b), the pressure $(\mathrm{Pa})$ is plotted on the front surface by fixing angle at $\pi / 6$ radians with $\kappa=1$ and the increasing Reynolds number. The plot shows that the enhancement in the Reynolds number produces a high value of the pressure with more negative pressure on the surface of the obstacle. In Figure 5(c), the impact has been checked by fixing the angle at $\pi / 6$ radians and with the initial value of $\operatorname{Re}=1000$. We come to know that even by increasing the volume of the pores in the screen, the enhancement in the pressure does not take place. Thus, optimization of the pressure on the surface of any object is functioned vertically and gradually optimized with the increment of the Reynolds number.

In the industrial area, measuring and controlling the temperature of the object do matter in the case when the nonisothermal flow has been observed by the attachment of the screen. In the current problem, the distribution of the temperature over the circular surface is given by the virtue of three parameter angles of the screen, nondimensional number $\mathrm{Re}$, and resistance of the screen. The pattern of the temperature over the circular surface has been checked by fixing any two of the three parameters. In Figures 6(a)-6(c), the distribution of the temperature over the surface of the obstacle has been presented through the graphs. It is clear from the graphs that the distribution of the temperature on the surface is almost nonlinear and unpredicted and changing from a minimum temperature of $T=293.5 \mathrm{~K}$ to the maximum temperature of $T=298.5 \mathrm{~K}$ from the initial temperature of $T_{\text {in }}=293.15$ which is allowed to enter from the inlet along with the air with initial velocity depending on the Reynolds number.
In Figure 6(a), the distribution of the temperature is detailed by changing the angle of the screen for $\pi / 6, \pi / 4$, and $\pi / 3$ radians at the constant Reynolds number of 1000 and the initial resistance $\kappa=1$. The graph shows that the values of the temperature on the surface are always greater for angle $\pi / 3$ degree for fixing the Reynolds number with the resistance coefficient $\kappa$. The temperature distribution over the surface is decreasing with the increase in the Reynolds number by fixing the orientation and resistance of the screen (see Figure 6(b)). The surface can get the maximum temperature at $\mathrm{Re}=1000$ and the minimum temperature at $\operatorname{Re}=10,000$. Changing the resistance value for keeping the other parameters constant does not give a significant change in the temperature of the surface (see Figure 6(c)).

The heat transfer coefficient can be defined as the heat energy transmitted per unit area per unit change in the temperature. It is used to measure the capability of the conductor to conduct the heat while any fluid is coming into contact with the solid at a certain temperature. The higher the heat transfer coefficient, the greater capability of the solid to conduct the heat with the convection process. In Figures $7(\mathrm{a})-7(\mathrm{c})$, the heat transfer coefficient is calculated at the front of the circular obstacle. The heat transfer coefficient is decreasing continuously down the surface which means that the convection process is weakened down the surface of the obstacle. By fixing $\operatorname{Re}=1000$ and $\kappa=1$, the heat transfer coefficient is decreasing with the same rate for all angles under selection. The phase transition is low for the angle of $\pi / 3$ radians and high for the angle of $\pi / 6$ radians (see Figure $7(\mathrm{a})$ ). The heat transfer coefficient shows a positive relationship with the increment of the Reynolds number and made the process of convection fast (see Figure 7(b)). We say for a particular position $y$ on the circular obstacle the convection process is improving about $8.33 \%$. In Figure $7(\mathrm{c})$, 


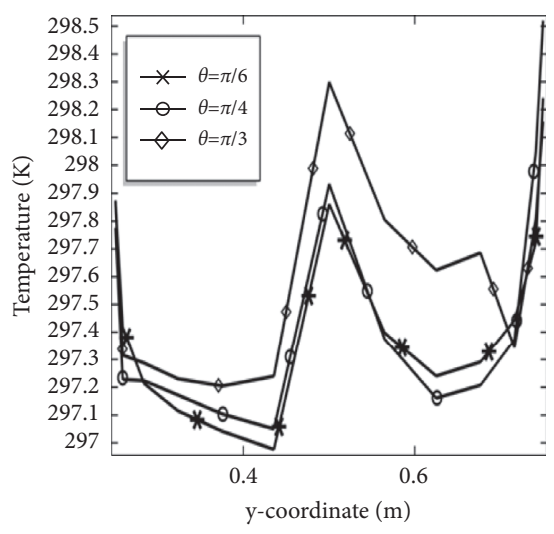

(a)



(b)

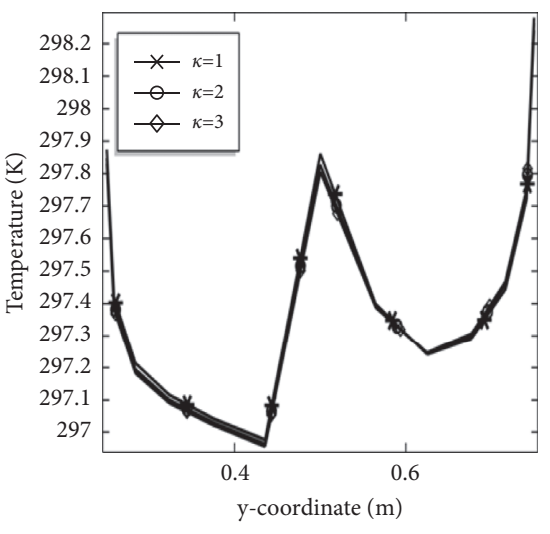

(c)

Figure 6: Temperature K on the front surface of the circular cylinder (a) for all angles by fixing $\operatorname{Re}=1000$ and $\kappa=1$; (b) for all Re by fixing $\kappa=1$ and $\theta=\pi / 6$; (c) for all $\kappa$ by $\operatorname{Re}=1000$ and $\theta=\pi / 6$.



(a)

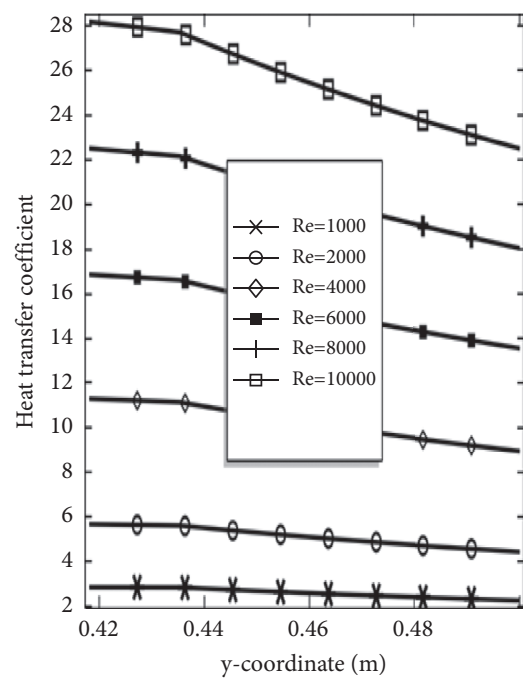

(b)

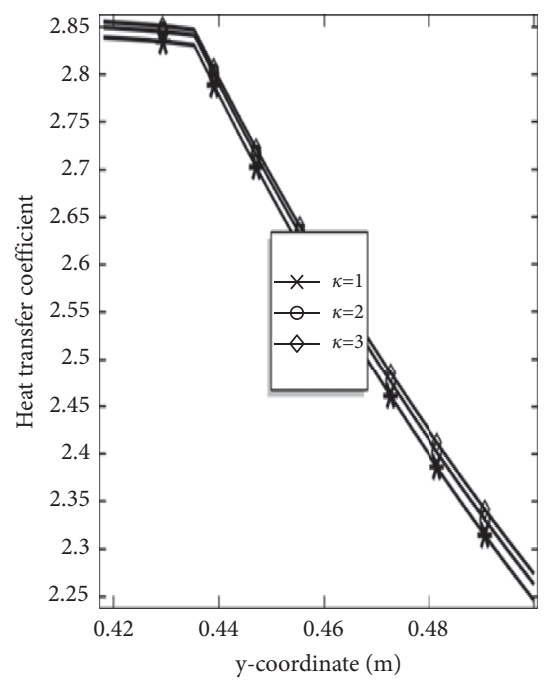

(c)

FiguRE 7: Heat transfer coefficient on the front surface of the circular cylinder (a) for all angles by fixing $\operatorname{Re}=1000$ and $\kappa=1$; (b) for all Re by fixing $\kappa=1$ and $\theta=\pi / 6$; (c) for all $\kappa$ by $\operatorname{Re}=1000$ and $\theta=\pi / 6$.

the heat transfer coefficient is expressed on the front surface of the circular obstacle by fixing $\mathrm{Re}=1000$ and an angle of $\pi / 6$ radians. The heat transfer coefficient shows an insignificant change with altering the resistance coefficient $\kappa$ of the screen. For a particular resistance, it is decreasing by about $26.6 \%$ in the front surface of the circular obstacle.

In any problem of the fluid flow via the heat transfer, the conduction and the convection process is occurring at the same time. The ratio between the convective processes of heat transfer to the conductive process is known as the Nusselt number, and it is nondimensional. It is known for a laminar flow that the Nusselt number is less than 100, but for the turbulent flow, the Nusselt number is ranging between 100 to 1000. Moreover, if the Nusselt number is increasing, it indicates that the convective process is faster than the conductive one and vice versa. The calculation of the local Nusselt number is presented in Figures 8(a)-8(c) by fixing any two of the three parameters. The local Nusselt number is decreasing continuously on the front surface of the circular obstacle indicating the domination of the conductive process. In Figure 8(a), the impact of the Nusselt number has been checked by altering the angle of the screen. The graph indicates altering the angle at $\pi / 6$ radians; the convective process may be faster than in the case of other angles. On the whole, the rate of decreasing heat transfer is nearly the same at all angles. In Figure 8(b), the local Nusselt numbers are calculated with the increase in the Reynolds number from 1000 to 10,000 showing that the local Nusselt is increasing Reynolds number. The rate of decrement is different for all the Reynolds numbers, but the higher the Reynolds number, the higher the rate of decrement. Finally, the local Nusselt number has been checked by altering the resistance coefficient $\kappa$ with the constant Re and the angle of the screen (see Figure 8(c)). The graph shows that there will be no notable improvement in the pattern of the Nusselt number that results in the domination of the conduction process over the convective process. 


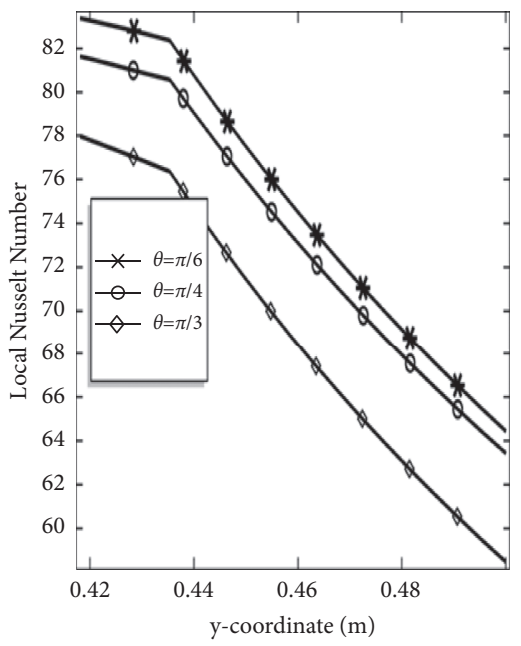

(a)

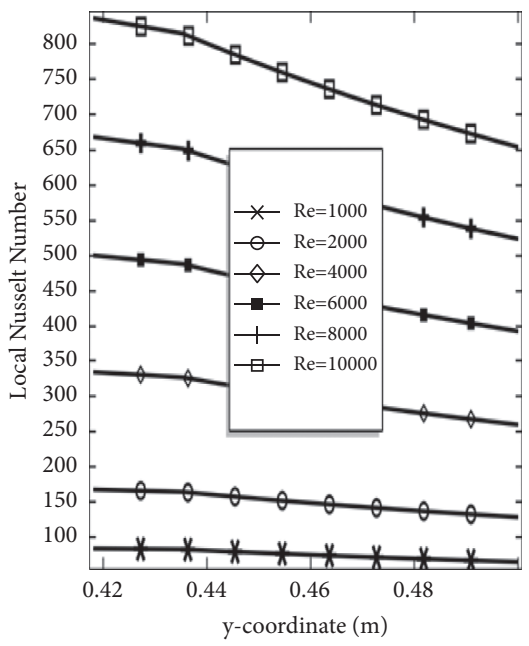

(b)

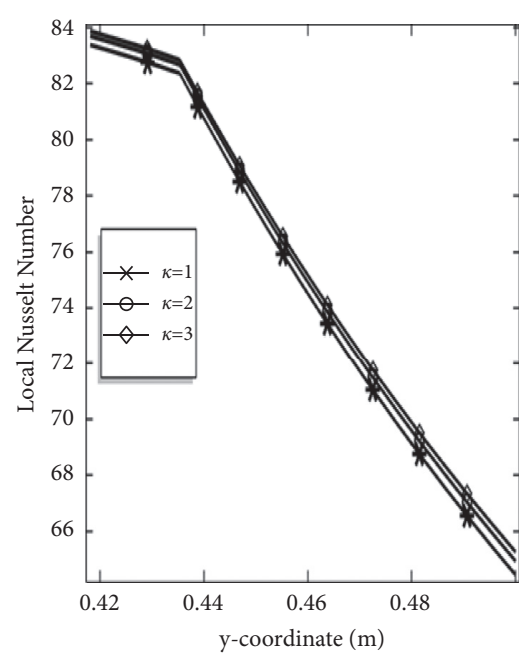

(c)

Figure 8: Evaluation of the local Nusselt number $(\mathrm{m} / \mathrm{sec})$ in the front surface of the circular cylinder (a) for all angles by fixing Re $=1000$ and $\kappa=1$; (b) for all Re by fixing $\kappa=1$ and $\theta=\pi / 6$; (c) for all $\kappa$ by $\operatorname{Re}=1000$ and $\theta=\pi / 6$.

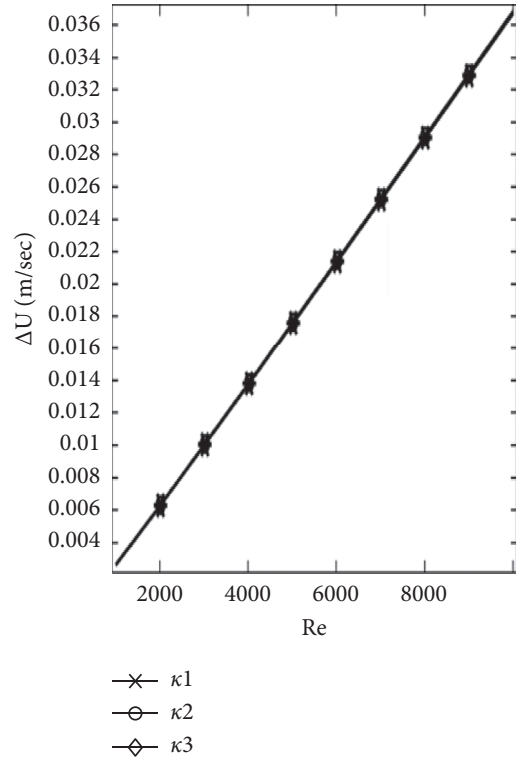

(a)

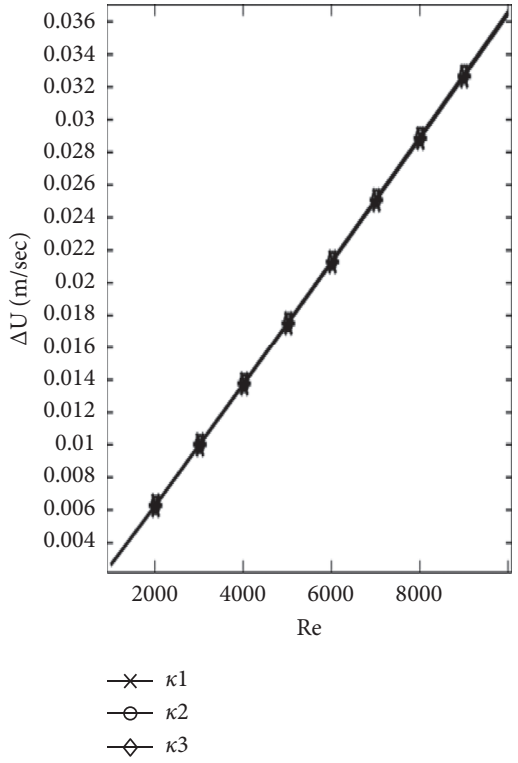

(b)

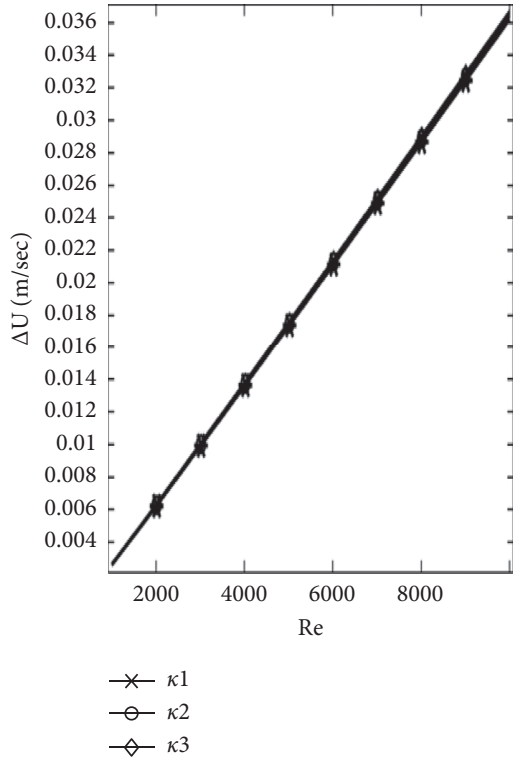

(c)

Figure 9: Rise of the velocity magnitude $(\mathrm{m} / \mathrm{sec})$ from inlet to the surface of the circular cylinder for all resistance coefficients: $(\mathrm{a}) \theta=\pi / 6$; (b) $\theta=\pi / 4$; (c) $\theta=\pi / 3$.

The testing fluid depending on the Reynolds number $(\mathrm{Re})$ is allowed to flow through the inlet of the channel and comes into the strike with the front surface of the channel. Here, we are going to present the difference in average velocity on the front surface and the initial velocity $U_{\text {in }}$. Figures 9(a)-9(c) present the calculation of the velocity change $\Delta U$ against Re, showing that the change in the flow rate of the particle is increasing linearly with the functions of the Reynolds number for all the angles $\pi / 6, \pi / 4$, and $\pi / 3$ radians with the same rate. Thus, to apply the linear regression process, we can determine the linear regression line that results in a linear equation connecting between the change in velocity and the Reynolds number, i.e., $\Delta U=3.86 e^{-6} \operatorname{Re}-0.0014$.

The difference of the average temperature in the surface of the circular obstacle and the inlet temperature Tin is presented in Figures 10(a)-10(c) for fixing the angle of the screen for all resistance $\kappa$ under the consideration. The difference is continuously decreasing with the increase in the Reynolds number for all angles with the same range. The decrease in the difference indicates that the average temperature is increasing on the front surface of the circular cylinder. The graph can be estimated through quadratic regression. Here, we are applying the quadratic regression 


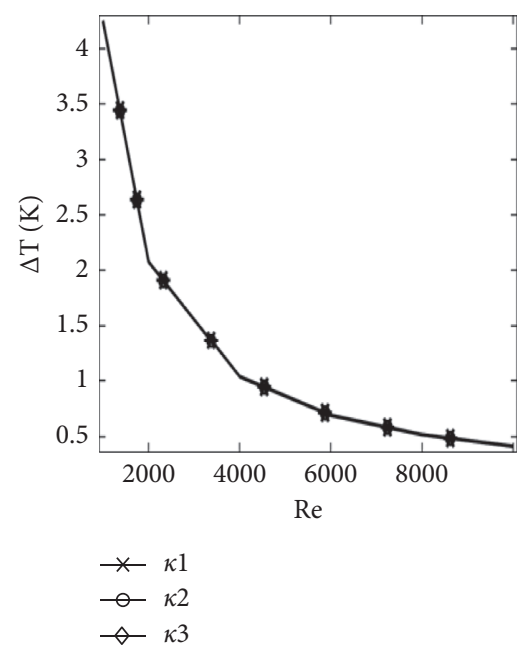

(a)

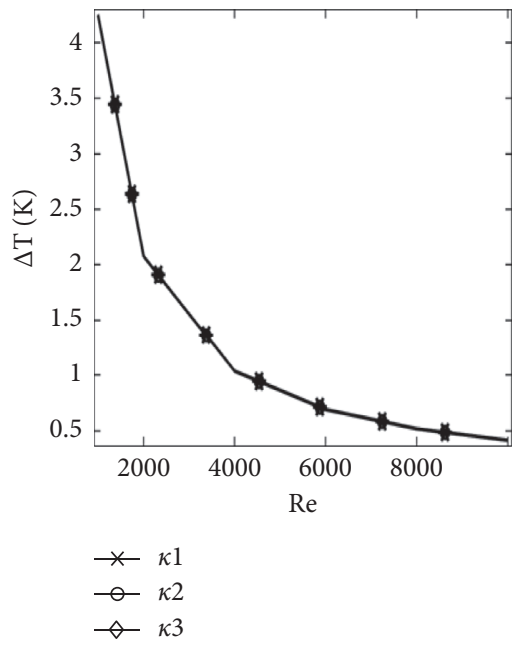

(b)

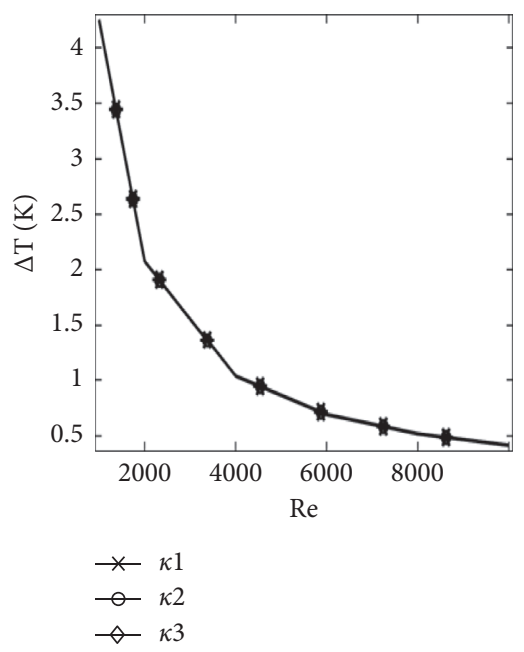

(c)

FiguRE 10: Rise of the temperature from inlet to the surface of the circular cylinder for all resistance coefficients: (a) $\theta=\pi / 6$; (b) $\theta=\pi / 4$; (c) $\theta=\pi / 3$.

estimation to get the nonlinear equation that relates the change in the temperature and the Reynolds number.

\section{Conclusion}

In the current simulation, a laminar, Newtonian fluid flow along with the heat transfer was observed through the rectangular channel with the screen affixed with the circular obstacle yielding the ratio of $1: 1$ to the height and diameter of the circle. The whole process was carried out through COMSOL Multiphysics 5.4 which adopts Galerkin's least square scheme to solve a coupled system formed by the twodimensional incompressible Navier-Stokes and heat equations. A parametric study was done by with the range of $1000 \operatorname{Re} 10,000$ and $\pi / 6 \leq \theta \leq \pi / 3$ and the resistance coefficient $1 \leq \kappa \leq 3$. The results are presented by the graph plots by fixing any two parameters out of three. The results were verified with the use of a correlation involving the inlet velocity, the current speed of the flow, and resistance and angle of the screen. In comparison, we found that we have achieved reliable results and we move our research forward by presenting new numerical results. The velocity magnitude, pressure, temperature, heat transfer, and the local Nusselt number are calculated on the front surface of the circular obstacle. In addition, the rise of the velocity magnitude and temperature from the inlet to the surface of the obstacle was discussed in terms of the Reynolds number. We have made the following conclusion points:

(1) The velocity magnitude on the front surface of the obstacle is linearly decreasing down the surface and increasing above the surface of the obstacle. Also, by altering the angles of the screen and the resistance coefficient $\kappa$ of the screen, there will be no significant change in the pattern of the velocity field. With the increase in the Reynolds number, the velocity magnitude on front of the surface is increasing but moves with the same pattern above and below the surface.

(2) In the case of pressure, it is increasing down the surface and increasing above the surface of the obstacle. The pressure is the function of the $y$-axis and behaves as a parabolic path in the front surface of the obstacle. With the change in the angle of the screen and the resistance coefficient, there will be no expressible change in the pattern of the value of the pressure; however, positive and negative pressure occurs at the same time above and below the obstacle. With the increase in the Reynolds number, the more negative value pressure is created above and down the surface of the obstacle.

(3) The temperature distribution of the front surface of the circular obstacle in the front surface is nonlinear with unpredictable directions. There will be no significant change by altering the angle of the screen as well as the resistance coefficient $\kappa$ of the screen. Although with the increase in the Reynolds number, the temperature of the object is decreasing significantly.

(4) The local heat transfer coefficient is decreasing continuously for all the angles of the screen with the same rate, but the heat transfer is maximum at the angle of $\pi / 6$ radians. The heat transfer coefficient is improving with the increase in the Reynolds number by fixing the angle and resistance provided by the screen. However, with the constant Reynolds number and the screen angle and with the altering of the resistance, there will be no improvement in the heat transfer coefficient. We can generally interpret that the convection process is declining from down to above the surface of the obstacle.

(5) The local Nusselt number is declining continuously from down to above the surface of the circle by fixing 
any two of the three parameters. This means that the conduction process is occurring faster than the convection process. With the increase in the Reynolds number, the value of the Nusselt number is increasing, and after $\operatorname{Re}=1000$, the fluid will become transit into the turbulent.

(6) The change in the velocity from the inlet to the surface of the circular obstacle is increasing linearly with the increase in the Reynolds number for all the values of the resistance. The increment is identical for all the angles under observation. The change in the temperature from the inlet to the surface of the obstacle is decreasing nonlinearly against the increase in the Reynolds number for all the resistance $\kappa$ of the screen under the observation. The characteristic of the declining temperature indicates that the average temperature on the surface of the object is increasing.

\section{Data Availability}

No data were used to support this study.

\section{Conflicts of Interest}

The authors declare that there are no conflicts of interest regarding the publication of this paper.

\section{Acknowledgments}

This research was funded by the Deanship of Scientific Research at Princess Nourah bint Abdulrahman University through the Fast-track Research Funding Program.

\section{References}

[1] T. R. Bott, Fouling of Heat Exchangers, Elsevier, Amsterdam, Netherlands, 1995.

[2] S. Jogee, B. V. S. S. S. Prasad, and K. Anupindi, "Large-eddy simulation of non-isothermal flow over a circular cylinder," International Journal of Heat and Mass Transfer, vol. 151, Article ID 119426, 2020.

[3] S. Jogee, K. Anupindi, and B. V. S. S. S. Prasad, "Large-eddy simulation of flow over three side by side circular cylinders," in Proceedings of the 8th International and 47th National Conference on Fluid Mechanics and Fluid Power, Guwahati, India, February 2021.

[4] P. Barnoon, D. Toghraie, and A. Karimipour, "Application of rotating circular obstacles in improving ferrofluid heat transfer in an enclosure saturated with porous medium subjected to a magnetic field," Journal of Thermal Analysis and Calorimetry, vol. 145, no. 6, pp. 3301-3323, 2021.

[5] M. Jawad, A. Saeed, A. Tassaddiq et al., "Insight into the dynamics of second-grade hybrid radiative nanofluid flow within the boundary layer subject to Lorentz force," Scientific Reports, vol. 11, no. 1, pp. 1-14, 2021.

[6] F. S. Swdi and M. Y. Jabbar, "Thermal analysis of nanofluid saturated in a semicircular hot enclosure cooled by a rotating half-immersed active circular cylinder subject to a convective condition," Heat Transfer, 2021.

[7] M. J. Haider, S. N. Danish, W. A. Khan, S. U. Mehdi, and B. A. Abbasi, "Heat transfer and fluid flow over circular cylinders in cross flow," NUST Journal of Engineering Sciences, vol. 3, no. 1, pp. 67-77, 2010.

[8] J. M. Park, O. J. Kim, S. J. Kim, and Y. C. Shin, "Heat transfer characteristics of circular and elliptic cylinders in cross flow," Advances in Mechanical Engineering, vol. 7, no. 11, Article ID $1687814015619553,2015$.

[9] X. Sun, Z. Ye, J. Li, K. Wen, and H. Tian, "Forced convection heat transfer from a circular cylinder with a flexible fin," International Journal of Heat and Mass Transfer, vol. 128, pp. 319-334, 2019.

[10] S. K. Singh, M. Kumar, A. Kumar, A. Gautam, and S. Chamoli, "Thermal and friction characteristics of a circular tube fitted with perforated hollow circular cylinder inserts," Applied Thermal Engineering, vol. 130, pp. 230-241, 2018.

[11] M. Abdelhady and D. Wood, "Effect of thermal boundary condition on forced convection from circular cylinders," Numerical Heat Transfer, Part A: Applications, vol. 76, no. 6, pp. 420-437, 2019.

[12] H. Ma and Z. Duan, "Similarities of flow and heat transfer around a circular cylinder," Symmetry, vol. 12, no. 4, p. 658, 2020.

[13] A. Subba Rao, C. H. Amanulla, N. Nagendra, O. Anwar Bég, and A. Kadir, "Hydromagnetic flow and heat transfer in a Williamson Non-Newtonian fluid from a Horizontal circular cylinder with Newtonian Heating," International Journal of Algorithms, Computing and Mathematics, vol. 3, no. 4, pp. 3389-3409, 2017.

[14] B. V. D. H. Zijnen, "Modified correlation formulae for the heat transfers by natural and by forced convection from horizontal cylinders," Applied Scientific Research, Section A, vol. 6, no. 2-3, pp. 129-140, 1956.

[15] W. Khan, R. Culham, and M. Yovanovich, "Fluid flow and heat transfer from a pin fin: analytical approach," in Proceedings of the 41st Aerospace Sciences Meeting and Exhibit, p. 163, Reno, Nevada, January 2003.

[16] W. A. Khan, J. R. Culham, and M. M. Yovanovich, "Fluid flow around and heat transfer from an infinite circular cylinder," Journal of Heat Transfer, vol. 127, no. 7, pp. 785-790, 2005.

[17] T. Igarashi, "Characteristics of a flow around two circular cylinders arranged in tandem: 1st report," Bulletin of JSME, vol. 24 , no. 188 , pp. 323-331, 1981.

[18] T. Igarashi, "Characteristics of a flow around two circular cylinders of different diameters arranged in tandem," Bulletin of JSME, vol. 25, no. 201, pp. 349-357, 1982.

[19] E. Buyruk, "Heat transfer and flow structures around circular cylinders in cross-flow," Turkish Journal of Engineering and Environmental Sciences, vol. 23, no. 5, pp. 299-316, 1999.

[20] M. A. Mehrabian, "Heat transfer and pressure drop characteristics of cross flow of air over a circular tube in isolation and/or in a tube bank," Arabian Journal for Science and Engineering, vol. 32, no. 2B, pp. 365-376, 2007.

[21] K. Szczepanik, A. Ooi, L. Aye, and G. Rosengarten, "A numerical study of heat transfer from a cylinder in cross flow," in Proceedings of the 15th Australasian Fluid Mechanics Conference, pp. 13-17, Sydney, Australia, December 2004.

[22] A. A. Memon, M. A. Memon, K. Bhatti et al., "Analysis of power law fluids and the heat distribution on a facing surface of a circular cylinder embedded in rectangular channel fixed with screen: a finite element's analysis," IEEE Access, vol. 9, pp. 74719-74728, 2021.

[23] I. Khan, A. A. Memon, M. A. Memon et al., "Finite element least square technique for Newtonian fluid flow through a semicircular cylinder of recirculating region via COMSOL multiphysics," Journal of Mathematics, vol. 2020, Article ID 8869308, 11 pages, 2020. 
[24] S. Rashidi, M. Bovand, and J. Abolfazli Esfahani, "Application of magnetohydrodynamics for suppressing the fluctuations in the unsteady flow around two side-by-side circular obstacles," The European Physical Journal Plus, vol. 131, no. 12, p. 423, 2016.

[25] F. Hammami, B. Souayeh, N. Ben-Cheikh, and B. Ben-Beya, "Computational analysis of fluid flow due to a two-sided lid driven cavity with a circular cylinder," Computers \& Fluids, vol. 156, pp. 317-328, 2017.

[26] R. Golani and A. K. Dhiman, "Fluid flow and heat transfer across a circular cylinder in the unsteady flow regime," International Journal of Engineering Sciences, vol. 3, no. 3, pp. 8-19, 2004.

[27] G. Hauke and T. Hughes, "A unified approach to compressible and incompressible flows," Computer Methods in Applied Mechanics and Engineering, vol. 113, no. 3-4, pp. 389-395, 1994.

[28] J. W. Elder, "Steady flow through non-uniform gauzes of arbitrary shape," Journal of Fluid Mechanics, vol. 5, no. 3, pp. 355-368, 1959.

[29] A. A. Memon, H. Shaikh, and A. A. Memon, "Finite element's analysis of fluid flow through the rectangular channel with inclined screens settled at angles," in Proceedings of the 2019 2nd International Conference on Computing, Mathematics and Engineering Technologies (iCoMET), pp. 1-5, IEEE, Sukur, Pakistan, January 2019.

[30] A. A. Memon, M. A. Memon, K. Bhatti, and G. M. Shaikh, "Finite element simulation of Newtonian and non-Newtonian fluid through the parallel plates affixed with single screen," European Journal of Pure and Applied Mathematics, vol. 13, no. 1, pp. 69-83, 2020.

[31] A. A. Memon, "Analysis of optimum velocity and pressure of the air flow through the screens with the help of resistance coefficient," Sukkur IBA Journal of Computing and Mathematical Sciences, vol. 3, no. 1, pp. 51-57, 2019.

[32] X.-Y. Gao, Y.-J. Guo, and W.-R. Shan, "Water-wave symbolic computation for the Earth, Enceladus and Titan: the higherorder Boussinesq-Burgers system, auto- and non-autoBäcklund transformations," Applied Mathematics Letters, vol. 104, Article ID 106170, 2020.

[33] X.-Y. Gao, Y.-J. Guo, and W.-R. Shan, "Hetero-Bäcklund transformation and similarity reduction of an extended $(2+1)$ dimensional coupled Burgers system in fluid mechanics," Physics Letters A, vol. 384, no. 31, Article ID 126788, 2020. 